\title{
EDITORIAL V. 7 N. 2 (2014)
}

ISSN: 1984-3151

\author{
Magali Maria de Araújo Barroso
}

\begin{abstract}
Doutora em Ciências em Engenharia de Sistemas e Computação. COPPE/UFRJ, 1987. Professora do Centro Universitário de Belo Horizonte - UniBH. Belo Horizonte, MG. magali.barroso@prof.unibh.br.
\end{abstract}

Para esta edição foram submetidos 36 artigos e 13 foram aceitos, sendo um deles, convidado. Todos utilizam as Ciências Exatas e a Tecnologia como ferramentas em seu desenvolvimento. Atualmente a revista possui Qualis B4 nas áreas de Engenharia II, Ensino, Geografia e de Interdisciplinaridade, Qualis B5 em Ciências Sociais Aplicadas I, além de Qualis C em Ciências Ambientais, Ciência da Computação, Ciência de Alimentos e Química.

Os artigos selecionados, após a análise dos conselhos Científico e Editorial e de Pareceristas ad hoc, versam sobre: Sustentabilidade, Engenharia Química, Química, Engenharia de Produção, Ciências Ambientais e Biotecnologia, Ciência da Computação e Geografia.

O artigo convidado foi desenvolvido pelos doutores: Rogério Alexandre Alves de Melo e Vitorio Delogo de Castro, respectivamente, em Engenharia Química pela UFSCar - São Carlos - SP e UFMG - Belo Horizonte - MG. O primeiro é professor da UFVJM Diamantina, MG e, o segundo, do UniBH - BH, MG.

ANÁlISE DA DESTINAÇÃO DO ÓLEO DE COZINHA RESIDUAL NA REgião oeste de Belo Horizonte/Minas Gerais e SUA TRANSFORMAÇÃO DE FORMA SUSTENTÁVEL faZ "uma análise de como é realizado o descarte inadequado do óleo de cozinha residual no entorno do Centro Universitário UNIBH-Campus Estoril, situado na cidade de Belo Horizonte-MG, visando ao desenvolvimento de projetos de extensão e de pesquisa sustentáveis para a sua minimização e transformação final através de um sólido catalítico. O procedimento metodológico inicial envolveu o cadastramento dos síndicos dos condomínios no entorno do UNIBH com consequente aplicação de questionário, possibilitando como resultado uma análise do destino e danos causados pelo óleo de cozinha residual quando descartado de forma inadequada nessa região. Com o intuito de minimizar os impactos ambientais os discentes dos cursos de Engenharia Química e Ambiental do UNIBH, desenvolveram projetos de extensão, visando à produção de Biodiesel e de produtos de limpeza para doações para Instituições carentes da região do entorno do UNIBH e também participaram, por dois anos seguidos, do evento "dia da responsabilidade social", promovido pelo Núcleo Acadêmico do UNIBH. Em outra linha de estudo, houve o preparo de um material sólido catalítico do tipo MCM-41 para utilização em reações avançadas, como a hidrogenólise do glicerol, produzindo o componente 1,2-propanodiol."

Os outros artigos são:

Análise da Centralidade da Ceasa minas em Relação aOS fluxos Hortigranjeiros em Minas GeraIS realizada por Sérgio Gontijo Faria e Antônio Carlos da Silva Souza, sendo, respectivamente, 
bacharel em Geografia pelo UniBH e Mestre em Geografia - Tratamento da Informação Espacila pela PUC Minas - Belo Horizonte - MG, também professor do UniBH - Belo Horizonte - MG. O trabalho estudo "a centralidade da Ceasa Minas em relação aos fluxos de hortigranjeiros no Estado de Minas Gerais, considerando $\mathrm{o}$ aumento da produção e o distanciamento das zonas produtoras. "

Avaliação do uso de resíduos siderúrgicos para DESCOLORAÇÃO DE EFLUENTES DE INDÚSTRIAS TÊXTEIS de autoria de Alisson Henrique Marques da Silva, Jorge David Alguiar Bellido, Maikel Laurence Maloncy e Marcelo da Silva Batista, sendo o primeiro mestre em Engenharia Química pela UFSCar - São Carlos SP, o segundo doutor pela USP - São Paulo - SP, o terceiro doutor pela The Hague University of Applied Sciences. The Hague, Holanda e, o ultimo, doutorpela UFSCar - São Paulo - SP, os três últimos professores da UFSJ - Outro Branco - MG. Eles avaliaram "o uso de resíduos industriais da laminação, têmpera e aciaria, para descoloração do corante azul de metileno em processo contínuo. Esses resíduos foram caracterizados por difratometria de raios $X(D R X)$ e avaliados como catalisadores na descoloração do azul de metileno. Os resultados de DRX mostraram a presença de hematita nos resíduos industriais. No processo contínuo de descoloração do corante azul de metileno, na presença de peróxido de hidrogênio e ácido, foi observada a descoloração da solução para os três resíduos. No entanto, a ordem de atividade dos resíduos foi: têmpera $(20 \%)$ < aciaria $(70 \%)$ < laminação (80\%). O resíduo de laminação também apresentou maior estabilidade catalítica nas condições da reação e mostrou-se promissor para o tratamento de efluentes de indústrias têxteis."

Avaliação dos padrões de identidade E de QUALIDADE DE AGUARDENTES DE FRUTAS desenvolvido por João Guilherme Pereira Mendonça, Maria das Graças Cardoso, Wilder Douglas Santiago, Leonardo Milani Avelar Rodrigues, Rodolfo Romaniello Cardoso e Rafaela Magalhães Brandão, sendo a segunda doutora em Química pela UFMG - Belo Horizonte, MG e os demais vinculados ao Curso de Química da UFLA - Lavras - MG. Anunciam que "A produção de aguardentes de frutas pode ser dividida em diferentes etapas, similares à produção da cachaça. As características marcantes nestas aguardentes são o sabor e o aroma típicos de cada fruta. Durante o processo de fabricação destas bebidas, ocorrem diversas reações químicas que proporcionam a incorporação de grande variedade de compostos a essa, sendo responsáveis junto a todo o processo pela qualidade da bebida. Dentre estes compostos, citam-se os fenólicos, que atuam como antioxidantes, combatendo os radicais livres, evitando diferentes doenças, sendo benéficos para saúde. Na produção de aguardente, alguns contaminantes podem ser formados. Entre estes se cita o carbamato de etila (CE), que vem sendo amplamente estudado devido aos seus efeitos tóxicos e carcinogênico. Este trabalho teve como objetivos avaliar a qualidade físico-química e determinar a composição fenólica total, a atividade antioxidante e a concentração de CE de três aguardentes de frutas. Constatou-se que os parâmetros físico-químicos analisados e $\quad \mathrm{O} \quad \mathrm{CE}$ estavam dentro dos limites estabelecidos pelo Ministério da Agricultura, Pecuária e Abastecimento (MAPA), e que cada bebida analisada apresentava teores de compostos fenólicos baixos em relação aos teores encontrados para as frutas quando comparados com dados da literatura."

Bacias, Problemas e Potencialidades: Estudo de Caso acerca do Baixo Curso da Bacla Hidrográfica do Córrego Bonsucesso, em Belo Horizonte, Minas Gerais elaborado por Flávio Aparecido da Silva, Hugo Patrick Oliveira Mendes, Leomar de Souza Moraes, Michael Jordan Goleme Silva, Rafael Lara Mazoni Andrade, Sérgio Augusto Ferreira, Silas Sidney Linhares Pinto e Taiza de Pinho Barroso Lucas, sendo os primeiros bacharelandos em 
Geografia e a última mestre em Geografia pela UFMG - Belo Horizonte - MG, todos vinculados ao UniBH Belo Horizonte - MG. O "artigo disserta acerca de conceitos atinentes a recursos hídricos, bacias hidrográficas e sua gestão. O trabalho mostra a imperiosa necessidade de se repensar 0 relacionamento entre seres humanos e recursos naturais. Utilizando uma pesquisa in loco aliada a uma metodologia de análise de observação, este artigo mostra problemas e alternativas de soluções para o córrego Bonsucesso, Belo Horizonte."

\section{Comparação do PERFIL FísICO-QUímICO dE CACHAÇAS} ENVELHECIDAS EM TONÉIS DE CARVALHO (QUERCUS SP) E AMBURANA (AMBURANA CEARENSIS) tem como autores um grupo de pesquisadores da UFLA - Lavras - MG, Wilder Douglas Santiago, Maria das Graças Cardoso, Juliana de Andrade Santiago, Leonardo Milani Avelar Rodrigues, Bruno Leuzinger da Silva e Alex Rodrigues Silva Caetano. São uma doutora, três doutorandos e dois graduandos, vinculados ao curso de Química. Tem como resumo: "A cachaça, tradicional e popular bebida brasileira, é o destilado mais consumido em nosso país. O conhecimento de sua composição química é importante, visto que os estudos dos compostos potencialmente tóxicos têm sido um fator determinante no controle de qualidade. $\mathrm{O}$ hábito do envelhecimento está se tornando uma prática comum entre os produtores que buscam agregar valores ao seu produto. Madeiras nativas vêm sendo usadas para o envelhecimento, substituindo a tradicional madeira de carvalho. O presente trabalho objetivou realizar um acompanhamento periódico da qualidade físicoquímica, quanto aos teores de grau alcoólico, acidez volátil, ésteres, aldeídos, álcoois superiores, furfural, metanol e cobre da cachaça no processo de produção e no envelhecimento em tonéis de carvalho (Quercus $\mathrm{sp}$ ) e amburana (Amburana cearensis). A bebida foi produzida em um alambique artesanal, situado no sul do Estado de Minas Gerais. As análises físicoquímicas foram realizadas de acordo com as especificações estabelecidas pelo Ministério de Agricultura, Pecuária e Abastecimento (MAPA). Pelos resultados, foi possível observar que as médias das frações do destilado em estudo apresentaram resultados diferenciados quanto à composição química e mantiveram essa diferença ao longo do período de envelhecimento da cachaça em ambos os tonéis de madeira em estudo."

CorrelaÇão entre EXTRATO SECO TOTAL, COMPOSIÇÃo FENÓLICA TOTAL E INTENSIDADE DE COR DE CACHAÇAS ENVELHECIDAS EM TONÉIS DE CARVALHO (QUERCUS SP) E amburana (AMBURANa CEARENSIS) EM UM PERÍOdO DE 12 MESES é de autoria de Wilder Douglas Santiago, Maria das Graças Cardoso, Marcos de Souza Gomes, Leonardo Milani Avelar Rodrigues, Rodolfo Romaniello Cardoso e Rafaela Magalhães Brandão, todos afiliados à UFLA - Lavras - MG, sendo uma doutora, três doutorandos e dois graduandos em Química ou Agroquímica. $\mathrm{O}$ artigo esclarece que "O envelhecimento é a última etapa do processo de produção de cachaça, não sendo obrigatória sua realização. É uma etapa importante na fabricação da cachaça, que possibilita ao produtor agregar valor em sua bebida. Durante o processo de envelhecimento, ocorrem tanto a extração de componentes da madeira pela cachaça, contribuindo para o desenvolvimento de uma cor diferenciada, como também o contato com o oxigênio atmosférico que entra pelos poros da madeira, favorecendo reações de oxidação dos componentes presentes na cachaça, conferindo à bebida características sensoriais extremamente distintas. Assim, os objetivos deste trabalho foram determinar e quantificar extrato seco total, composição fenólica total e intensidade de cor de cachaças envelhecidas em tonéis de carvalho e amburana em um período de 12 meses e averiguar possível correlação linear destes parâmetros analisados. De acordo com os resultados de extrato seco, composição fenólica total e intensidade de cor, constatou-se uma correlação linear positiva 
satisfatória entre eles em ambas as madeiras. As variações ocorridas no aumento de um dos parâmetros acarretam um aumento em outro ao longo do envelhecimento da bebida."

Desenvolvimento e anÁlise físico-química dA FARINHA DA CASCA, DA CASCA IN NATURA E DA POLPA DE BANANA VERDE DAS CULTIVARES MAÇÃ E PRATA escrito por Ludimila Gasparotto Castilho, Bruna Merigio Alcantara e Edmar Clemente, respectivamente, Bacharel em Engenharia de Alimentos, mestranda e doutor em Ciência de Alimentos pela UEM - Maringá - PR. Relatam que "A bananeira é cultivada em sua maioria em países tropicais. No Brasil, a banana perde apenas para a produção de laranjas e tem como maior produtor o estado da Bahia, sendo uma fruta de elevado valor nutricional. A casca apresenta teores de nutrientes maiores que a parte comestível e, por ser um resíduo, torna-se interessante seu aproveitamento com o intuito de minimizar perdas e desenvolver produtos que possam ser inseridos na alimentação humana, no caso a farinha da casca. $O$ presente trabalho tem como objetivo desenvolver e avaliar as características físico-químicas da farinha de casca de banana verde dos cultivares maçã e prata, bem como compará-las às da casca in natura e da polpa da fruta verde. As pencas foram adquiridas no comércio do município de Maringá e transportadas ao Laboratório de Bioquímica de Alimentos da Universidade Estadual de Maringá; foram lavadas, sanitizadas e descascadas. A casca foi cortada, submetida ao branqueamento, secada e triturada para obtenção da farinha. A polpa, a casca in natura e a farinha da casca de banana foram avaliadas quanto ao teor de: acidez titulável, $\mathrm{pH}$, açúcares redutores e totais, amido, minerais, umidade, proteínas e lipídeos. Os resultados foram analisados pelo teste de Tukey $(P \leq 0,05)$. As farinhas da casca dos dois cultivares apresentaram-se nutricionalmente superiores à polpa da fruta e à casca in natura. A umidade mostrou-se dentro do padrão permitido pela legislação de farinhas. De forma geral, a banana verde apresentou alto potencial nutricional para elaboração de farinha e sua aplicação na alimentação."

\section{Estudo da Alteração do Projeto de um Volante Convencional para Implantação de Airbag de} autoria de Jonas Paulino Rodrigues Jr., Lucas da Silveira Franco, Vinícius Finardi Zampieri, Gustavo Longhi de Carvalho e Juliano Schimiguel. Os três primeiros sõa bacharéis em Engenharia de Produção pela UNIANCIETA - Jundiaí - SP, o quarto é mestre em Engenharia Mecânica pela mesma universidade e o último é doutor em Ciência da Computação da UNICAMP - Campinas - SP e professor da UNIANCHIETA e da UNINOVE - São Paulo, SP. Consideram que "Diante da obrigatoriedade do uso de airbag em todos os veículos produzidos no Brasil a partir de 2014, este trabalho tem como objetivo demonstrar a importância do airbag como item de segurança, com a finalidade não de reduzir acidentes, mas sim de diminuir o índice de mortes em acidentes. Para atender à lei, a empresa foco do nosso trabalho modificou o projeto e o processo de fabricação de um volante convencional para uma versão airbag, buscando segurança, satisfação dos clientes e retorno financeiro. $O$ departamento de engenharia de desenvolvimento da empresa vem trabalhando para implantar o airbag sem grandes alterações no design requerido pelo cliente. Essas mudanças, que são objeto de estudo deste trabalho, indicaram que a engenharia da empresa projetou um volante, visando a atender ao mercado exterior, e utilizou parte deste projeto para adequar as modificações que estão projetadas para o mercado nacional."

\section{Prevenção e Contenção de Processos Erosivos} Causados Pela Expansão Urbana no Município de Belo Horizonte - Minas Gerais escrito por André Bicalho Luz, Bárbara Carolina de Oliveira Passos, Bianca de Castro Sampaio, Eduardo Teixeira Gregório, Jeisiane Rodrigues Pinto, João Cláudio 
Dimeira, Juliana Pinheiro Gonçalves, Nayla Izabel Souza Moreira e Camila Moreira de Assis, sendo os primeiroa bacharelando em Engenharia Ambiental no UniBH - Belo Horizonte - MG e a última doutora pela UFMG e professora do UniBH. O resumo do artigo diz: "Um processo erosivo ocorrente na Região Oeste de Belo Horizonte, no bairro Buritis, foi analisado a fim de se descobrir se a urbanização o afetaria, além de propor medidas de prevenção e sua contenção. Este foi denominado como ravina; realizaram-se análises morfológicas do solo para que seu tipo e horizontes fossem estabelecidos, além de indicar se o solo era susceptível à erosão. Através de uma análise estatística, descobriu-se uma relação entre as percentagens de alguns componentes do solo. Verificou-se que a área em estudo, com o auxílio da declividade, do tipo de solo e do alto nível de urbanização possui alta susceptibilidade a sofrer processos erosivos. Um método de prevenção seria a não ocupação da área, visto sua alta declividade e o tipo de litologia extremamente friável. Os métodos de contenção mais indicados para a área seriam a revegetação do local em conjunto com as paliçadas. As plantas mais indicadas são Leucena (Leucaena leucocephala), Puerária (Pueraria phaseoloides) e Sesbânea (Sesbania virgata), que são de portes arbustivo-arbóreas, podem ser utilizadas em consórcio com as gramíneas. É importante esclarecer que a recuperação de qualquer área deve ser realizada através de muitos estudos e por uma equipe técnica multidisciplinar, em que os aspectos social, econômico e ambiental sejam considerados na tomada de decisões."

Proposta de um Esquema data Warehouse de REFERÊNCIA PARA SISTEMAS ENTERPRISE RESOURCE Planning (ERP): Módulos Financeiro e Contábil de autoria de Diogo Souza de Figueiredo, Myrian Gabriela Alves Camilo e Antônio da Mota Moura Junior, sendo os dois primeiros bacharéis em Ciência da Computação pelo UniBH - Belo horizonte, MG e, o último, mestre em Tecnologia da Informação pela Fundação João Pinheiro - Belo Horizonte, MG e professor do UniBH. Trabalham com Banco de Dados e reconhecem que "As necessidades de informações para tomada de decisão ocorrem em qualquer tipo de empresa. Os sistemas integrados de gestão empresarial (Enterprise Resourse Plannig - ERP) aparecem como um dos principais focos de investimentos relacionados à tecnologia de informação nas empresas, porém, para obter relatórios com rapidez do seu fluxo de caixa, de certa forma torna-se incompatível com a estrutura básica dos seus diversos módulos. Assim este trabalho apresenta uma proposta de Data Warehouse (DW) para gerar informações gerenciais dos módulos ERP financeiro e contábil. Como resultado, apresenta um modelo dimensional como proposta de tomada de decisão."

\section{Seleção de amostras de AspergilluUs SP isoladas} da CaAtinga de Pernambuco e produção de Ácido CÍTRICO POR FERMENTAÇÃO SUBMERSA, pesquisa desenvolvida por Márcia Caetano de Sá Muniz, Brindize Ferreira de Lima, Galba Maria de Campos Takaki e Carlos Alberto Alves da Silva, sendo as duas primeiras, respectivamente, mestranda e mestre em Desenvolvimento de Processos Ambientais. Os dois últimos são doutores, ele em Microbiologia e ela em Biotecnologia, todos vinculados à ÚNICAP - Recife, PE. Atestam que "O isolamento de novas espécies microbianas em regiões como a Caatinga nordestina tem direcionado novos estudos para a identificação de novas espécies e gêneros microbianos, realização de ensaios biotecnológicos voltados para produção de metabólitos de alto valor agregado. O ácido cítrico é um ácido orgânico comum de quase todos os seres vivos, presente naturalmente em frutas cítricas e apresenta vasta aplicabilidade comercial. Sua produção via micro-organismos é realizada por espécies capazes de converter carboidratos em ácido cítrico, cuja produção é fortemente influenciada pela natureza dos substratos presentes nos meios de 
fermentação, pois alguns micronutrientes são de extrema importância para a fixação biológica, transformando-se em co-fatores na incorporação de nitrogênio e ocasionando uma melhor produção do bioproduto. O gênero Aspergillus tem se destacado como um bom produtor de ácido cítrico e de outros metabólitos de interesse biotecnológico. Foram realizados estudos de seleção de três amostras de Aspergillus $\mathrm{sp}$ isoladas da Caatinga do Estado de Pernambuco, denominadas de SIS 09, 10 e 16 para produção de ácido cítrico em meio sólido e produção de ácido cítrico através de fermentação submersa com a melhor amostra selecionada. Foram testados três meios com diferentes composições. Os experimentos foram realizados em shaker orbital a $28^{\circ} \mathrm{C}, 150 \mathrm{rpm}$, durante 144 horas. Foram coletadas amostras a cada 24 horas para determinações da presença de $\mathrm{AC}, \mathrm{pH}$ e açúcares redutores totais. Os resultados obtidos evidenciaram que, nos ensaios de seleção de produção de ácido cítrico em meio sólido, a amostra SIS 09 apresentou a formação do maior halo característico obtido de $3,0 \mathrm{~cm}$. Nos ensaios de produção através de fermentação submersa, o meio denominado $M_{1}$ apresentou uma produção de ácido cítrico de 3,05 g/L, pH final de 5,0. A seleção de novos micro-organismos produtores de moléculas potencialmente ativas induz a realização de novos estudos biotecnológicos para melhorarem as condições de produção dos bioprodutos desejados."

Uma Abordagem sobre Problemas de Segurança DA INFORMAÇÃO POR MEIO DO DESENVOLVIMENTO DE APLICAÇões MALICIOSAS PARA ANDROID desenvolvido por Leandro Rodrigues Inácio e Antônio Ricardo Leocádio Gomes, respectivamente, bacharel em Ciência da Computação e Especialista em Novas tecnologias em Educação e Treinamento, ambos vinculados ao UniBH - Belo Horizonte - MG. O "artigo discute o desenvolvimento de aplicações maliciosas e o nível de conhecimento do usuário sobre segurança do Sistema Operacional móvel Android. Este trabalho visa a demonstrar o nível de dificuldade envolvido no desenvolvimento de uma aplicação maliciosa até a fase de disseminação."

Esta edição contém artigos das cidades de Belo Horizonte, Diamantina, Lavras e Ouro Branco de Minas Gerais, Maringá do Paraná, Recife de Pernambuco e Jundiaí e São Paulo de São Paulo.

Nossos agradecimentos aos pesquisadores que contribuíram com artigos, aos conselhos Científico e Editorial e aos Pareceristas ad hoc, cujo trabalho de avaliação permitiram a publicação desta edição.

Informamos que a partir da próxima edição, após cinco anos de dedicação à Revista e-xacta como editora geral, teremos como sucessor Vitório Delogo de Castro, doutor em Química pela UFMG e professor do UniBH. Agradecemos a todos que contribuíram com artigos submetidos e publicados, durante a nossa passagem por este periódico, pela confiança depositada. Somos gratas, também, aos membros dos conselhos Editorial e Científico e aos Pareceristas ad hoc, parceiros de trabalho, sempre preocupados com a qualidade dos artigos. Aos diretores $e$ coordenadores do Instituto de Ciências e Tecnologia e à Administração Superior do UniBH e da Ânima, os nossos agradecimentos. Aos demais colegas, editores das revistas eletrônicas do UniBH, a certeza de que juntos formamos uma equipe séria $e$ atuante. Desejamos, ao novo editor, sucesso e muito trabalho, já que, este é o termômetro que mede o alcance da Revista e-xacta. 